\title{
Gradeability for Automobiles
}

\author{
Akilesh Yamsani ${ }^{1}$ \\ ${ }^{I}$ (Automobile Engineering, M.V.S.R Engineering College, India)
}

\begin{abstract}
The demand for transport has been growing rapidly and the footprints of roads have been widespread to areas which were previously inaccessible, especially hilly areas. Road inclinations are not always at zero. Vehicles should have the capability to take gradients even on full loads. The Gradeability of a vehicle is dependent on multiple factors such as the type of road surface, weight of the vehicle, tractive effort at the wheels, tire grip (at low speeds the aerodynamic drag is ignored). Since a vehicle should be ready to be driven on all types of terrains, tractive effort and tire grip become the deciding factors. This paper presents a method to determine the gradeability and enhance it to the required scale.
\end{abstract}

Keywords: gradeability, resistance, tractive effort, traction, traction diagram.

\section{INTRODUCTION}

Gradeability is defined as the highest grade a vehicle can ascend maintaining a particular speed. When designing an automobile, various performance targets are kept in view, the major ones being weight, fuel economy, power, torque, speed, and acceleration, gradeability is given a lesser significance unless a special purpose vehicle is being designed like a Sports Utility Vehicle (SUVs) or other heavy duty vehicles. Urban vehicles do have to encounter situations where they might have to ascend slopes like a basement or a bridge. These situations might prove to become difficult for the driver when on full load, hence making it an important performance factor not only for Special Purpose vehicles but also to those driven in the urban land.

Gradeability is measured in multiple ways, mainly:

- As an angle of inclination to the horizontal $\left(20^{\circ}, 30^{\circ}, 45^{\circ}\right.$, etc. $)$.

- As a percentage of rise over run.

- As a ratio of one part run to some particular number of parts (1 in 20 meters, 1 in 50 feet, etc.)

\section{APPROACH}

The major factors affecting the Gradeability were discussed concisely above, going to the mechanic of the problem, a vehicle is subjected to three resistances chiefly, and they are, rolling resistance, gradient resistance and aerodynamic resistance. If a vehicle has to start rolling, it has to generate enough tractive force at the wheels to exceed these resistances.

\section{$1.1 \quad$ Tractive force}

It is the net force available at wheels. To determine this force we use the formula:

$$
\frac{T_{e} \rho_{t} G}{R_{r}}(1)
$$

$\mathbf{T}_{\mathrm{e}}$ : Torque at the engine

$\boldsymbol{\rho}_{\mathrm{t}}$ : Transmission efficiency

G: gear ratio

$\mathbf{R}_{\mathbf{r}}$ : rolling radius

\subsection{Rolling resistance}

This is the resisting force that opposes the rolling of the tires, which is caused due to non-elastic effects at the tire-road surface; it is given by the formula:

$$
\boldsymbol{R}_{\boldsymbol{r}}=\boldsymbol{k} \boldsymbol{W} \cos \boldsymbol{\theta}(2)
$$

k: coefficient of rolling resistance (.2 for loose sand)

W: weight of the vehicle in $\mathrm{N}$

$\Theta$ : Angle of inclination

\subsection{Gradient resistance}

When negotiating a slope, a component of weight acts against the direction of motion which is proportional to the angle of inclination of the road surface. 


$$
\boldsymbol{R}_{\boldsymbol{g}}=\boldsymbol{W} \sin \theta(3)
$$

\subsection{Aerodynamic Resistance}

Similarly, we consider the resistance offered from the air in the direction opposite to motion of the vehicle:

$$
R_{d}=\frac{1}{2} \rho C_{d} A V^{2}(4)
$$

$\boldsymbol{\rho}$ : Density of air $\left(1.2 \mathrm{~kg} / \mathrm{m}^{3}\right)$

$\mathbf{C}_{\mathbf{d}}$ : Coefficient of drag ()

A: Projected area in $\mathrm{m}^{2}$

$\mathbf{V}$ : velocity in $\mathrm{m} / \mathrm{s}$

\subsection{Total Resistance}

$$
\mathbf{R}=\boldsymbol{R}_{\boldsymbol{r}}+\mathbf{R}_{\mathbf{g}}+\mathbf{R}_{\mathbf{d}}(5)
$$

\section{Case Study}

The above approach can be explained with a case study of an All-Terrain Vehicle designed for competing at SAE BAJA INDIA and we draft a Traction Diagram to estimate the Gradeability of this vehicle.

The specifications of the vehicle are as follows:

\begin{tabular}{|l|l|}
\hline \multicolumn{2}{|c|}{ Table 1 } \\
\hline Vehicle & BAJA SAE BUGGY \\
\hline Power & $10 \mathrm{hp} @ 3250 \mathrm{rpm}$ \\
\hline Torque & $19.66 \mathrm{~N}-\mathrm{m} @ 2800 \mathrm{rpm}$ \\
\hline Weight & $300 \mathrm{~kg}$ \\
\hline Tyre Radius & $10.5 \mathrm{inch} / .2667 \mathrm{~m}$ \\
\hline Rolling radius $\left(.96^{*} \mathrm{R}\right)$ & $.256 \mathrm{~m}$ \\
\hline Frontal projected area & $.7235 \mathrm{~m}^{2}$ \\
\hline Transmission efficiency & $85 \%$ \\
\hline Gearbox type & Sequential \\
\hline
\end{tabular}

Table 2

\begin{tabular}{|l|l|}
\hline Gear & Gear ratio \\
\hline 1 & $31.48: 1$ \\
\hline 2 & $18.70: 1$ \\
\hline 3 & $11.40: 1$ \\
\hline 4 & $7.66: 1$ \\
\hline Reverse & $55.08: 1$ \\
\hline
\end{tabular}

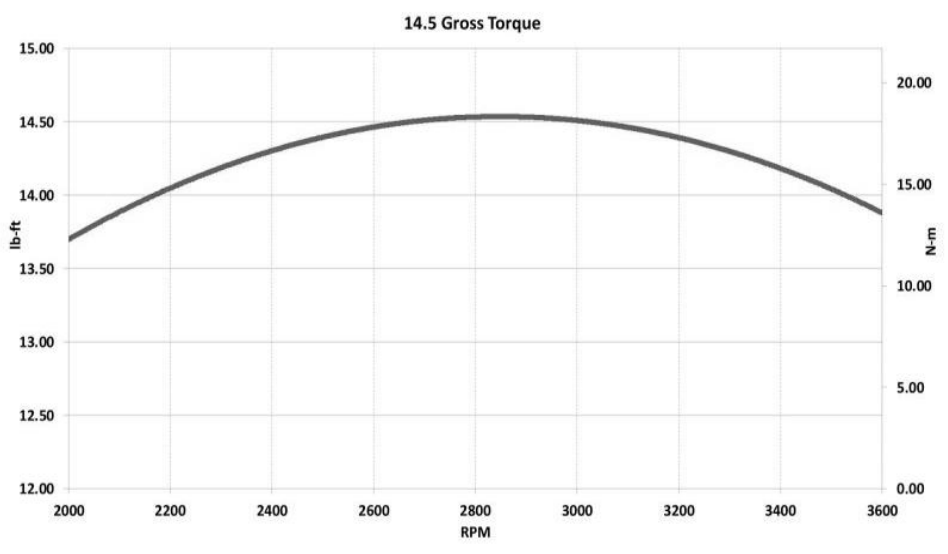

\subsection{Tractive effort calculations}

Fig. 1

Using equation (1) we calculate the tractive force at wheels for different gears. 
Table 3

\begin{tabular}{|c|c|}
\hline Gear & Tractive effort (N) \\
\hline 1 & 2055 \\
\hline 2 & 1220 \\
\hline 3 & 744 \\
\hline 4 & 500 \\
\hline$R$ & 3595 \\
\hline
\end{tabular}

\subsection{Calculations of different resistances}

\subsubsection{Rolling resistance}

The second step involves calculating the rolling resistance for different gradients, ranging from $0 \%$ to $100 \%$ using equation (2).

Table 4

\begin{tabular}{|c|c|}
\hline Gradient (\%) & $\mathbf{R}_{\mathbf{r}}$ \\
\hline 0 & 588.6 \\
\hline 20 & 577.5 \\
\hline 40 & 546.8 \\
\hline 60 & 504.7 \\
\hline 80 & 457.7 \\
\hline 100 & 416.42 \\
\hline
\end{tabular}

\subsubsection{Gradient resistance}

The third step is to calculate the gradient resistance for different grades by using equation (3).

Table 5

\begin{tabular}{|c|l|}
\hline Gradient (\%) & $\mathbf{R}_{\mathbf{g}}$ \\
\hline 0 & 0 \\
\hline 20 & 577.17 \\
\hline 40 & 1092.9 \\
\hline 60 & 1515.75 \\
\hline 80 & 1852.08 \\
\hline 100 & 2081.01 \\
\hline
\end{tabular}

\subsubsection{Air resistance}

The following table contains the drag force values Table 6

\begin{tabular}{|l|l|}
\hline Speed $(\mathbf{k m p h})$ & $\mathbf{R}_{\mathbf{d}}$ \\
\hline $\mathbf{5}$ & .629 \\
\hline 10 & 2.516 \\
\hline 15 & 5.652 \\
\hline 20 & 10.06 \\
\hline 25 & 15.726 \\
\hline 30 & 22.43 \\
\hline 35 & 30.76 \\
\hline 40 & 40.117 \\
\hline 45 & 50.87 \\
\hline 50 & 62.8 \\
\hline 55 & 76 \\
\hline
\end{tabular}

\subsubsection{Total resistance}

For this, we take the values of all the resistances obtained at a particular gradient for different speeds and add them to get the total resistance. We tabulate this data for drafting

\subsubsection{0\% Gradient}


Table 7

\begin{tabular}{|l|l|}
\hline Speed & Traction \\
\hline 0 & 588.6 \\
\hline 5 & 589.229 \\
\hline 10 & 591.116 \\
\hline 15 & 594.252 \\
\hline 20 & 598.66 \\
\hline 25 & 604.326 \\
\hline 30 & 611.03 \\
\hline 35 & 619.36 \\
\hline 40 & 628.71 \\
\hline 45 & 639.47 \\
\hline 50 & 651.4 \\
\hline 55 & 664.6 \\
\hline
\end{tabular}

\subsubsection{20\% Gradient}

Table 8

\begin{tabular}{|l|l|}
\hline Speed & Traction \\
\hline 0 & 1154.67 \\
\hline 5 & 1155.303 \\
\hline 10 & 1157.19 \\
\hline 15 & 1160.326 \\
\hline 20 & 1164.734 \\
\hline 25 & 1170.4 \\
\hline 30 & 1177.04 \\
\hline 35 & 1185.374 \\
\hline 40 & 1194.374 \\
\hline 45 & 1205.544 \\
\hline 50 & 1217.474 \\
\hline 55 & 1230.374 \\
\hline
\end{tabular}

\subsubsection{3 $40 \%$ Gradient}

Table 9

\begin{tabular}{|c|l|}
\hline Speed & Traction \\
\hline 0 & 1639.7 \\
\hline 5 & 1640.329 \\
\hline 10 & 1642.216 \\
\hline 15 & 1645.352 \\
\hline 20 & 1649.76 \\
\hline 25 & 1655.426 \\
\hline 30 & 1662.13 \\
\hline 35 & 1670.46 \\
\hline 40 & 1679.817 \\
\hline 45 & 1690.57 \\
\hline 50 & 1702.15 \\
\hline 55 & 1715.7 \\
\hline
\end{tabular}




\subsubsection{60\% Gradient}

Table 10

\begin{tabular}{|l|l|}
\hline Speed & Traction \\
\hline 0 & 2020.45 \\
\hline 5 & 2063.179 \\
\hline 10 & 2064.706 \\
\hline 15 & 2068.202 \\
\hline 20 & 2072.61 \\
\hline 25 & 2078.276 \\
\hline 30 & 2084.98 \\
\hline 35 & 2093.31 \\
\hline 40 & 2102.667 \\
\hline 45 & 2113.42 \\
\hline 50 & 2125.35 \\
\hline 55 & 2138.55 \\
\hline
\end{tabular}

1.7.4.5 80\% Gradient

Table 11

\begin{tabular}{|l|l|}
\hline Speed & Traction \\
\hline 0 & 2211.5 \\
\hline 5 & 2310.409 \\
\hline 10 & 2312.296 \\
\hline 15 & 2315.432 \\
\hline 20 & 2319.84 \\
\hline 25 & 2325.506 \\
\hline 30 & 2332.21 \\
\hline 35 & 2340.54 \\
\hline 40 & 2349.897 \\
\hline 45 & 2359.897 \\
\hline 50 & 2372.58 \\
\hline 55 & 2385.78 \\
\hline
\end{tabular}

\subsubsection{100\% Gradient:}

Table 12

\begin{tabular}{|l|l|}
\hline Speed & Traction \\
\hline 5 & 2498.054 \\
\hline 10 & 2499.946 \\
\hline 15 & 2503.082 \\
\hline 20 & 2507.49 \\
\hline 25 & 2513.156 \\
\hline 30 & 2519.86 \\
\hline 35 & 2528.19 \\
\hline 40 & 2537.54 \\
\hline 45 & 2548.3 \\
\hline 50 & 2560.23 \\
\hline 55 & 2573.43 \\
\hline
\end{tabular}




\subsection{Tractive force Available}

From the range of 2000-3600rpm the speed and traction available at different gears should be calculated. For this, the torque diagram (figure 1) for the engine should be referred to find the exact torque available at different intervals of engine speed.

For example, in first gear, at 2000rpm, the torque available at engine output is $13.7 \mathrm{~N}-\mathrm{m}$, by putting this in equation (1); we get the tractive force at the wheels.

Also, convert the angular velocity to linear velocity.

\subsubsection{First gear}

(Using the formula: $\frac{\pi \mathrm{DN}}{\mathrm{G}}$ ).

Table 13

\begin{tabular}{|l|l|}
\hline Speed & Traction \\
\hline 6.45 & 1941.41 \\
\hline 7.1 & 1991.058 \\
\hline 7.74 & 2026.491 \\
\hline 8.39 & 2047.605 \\
\hline 9.038 & 2065.374 \\
\hline 9.684 & 2047.605 \\
\hline 10.329 & 2040.602 \\
\hline 10.91 & 2012.067 \\
\hline 11.62 & 1962.732 \\
\hline
\end{tabular}

\subsubsection{Second gear}

Table 14

\begin{tabular}{|l|l|}
\hline Speed & Traction \\
\hline 10.846 & 1168.118 \\
\hline 11.93 & 1197.991 \\
\hline 13.0152 & 1219.311 \\
\hline 14.099 & 1232.015 \\
\hline 15.18 & 1242.706 \\
\hline 16.269 & 1232.015 \\
\hline 17.35 & 1227.801 \\
\hline 18.43 & 1210.632 \\
\hline 19.52 & 1180.948 \\
\hline
\end{tabular}

\subsubsection{Third gear}

Table 15

\begin{tabular}{|l|l|}
\hline Speed & Traction \\
\hline 17.9 & 703.025 \\
\hline 19.7 & 721.004 \\
\hline 21.49 & 733.83 \\
\hline 23.28 & 741.481 \\
\hline 25.07 & 747.916 \\
\hline 26.86 & 741.481 \\
\hline 28.65 & 738.945 \\
\hline 30.45 & 728.612 \\
\hline 32.24 & 710.747 \\
\hline
\end{tabular}

\subsubsection{Fourth gear}

Table 16

\begin{tabular}{|l|l|}
\hline Speed & Traction \\
\hline 26.656 & 472.33 \\
\hline 29.32 & 484.416 \\
\hline 31.98 & 493.036 \\
\hline 34.65 & 498.173 \\
\hline 37.318 & 502.496 \\
\hline 39.98 & 498.173 \\
\hline 42.64 & 496.469 \\
\hline
\end{tabular}




\begin{tabular}{|l|l|}
\hline 45.3152 & 489.527 \\
\hline 47.98 & 477.524 \\
\hline
\end{tabular}

\subsection{Traction diagram}

After the calculation of Tractive effort required and Tractive effort available are done and tabulated, the last step is to use this data to create a graph called Traction Diagram, which is a measure to check the climbing performance of an automobile. The Traction available and traction required are plotted as a function of the vehicle speed.

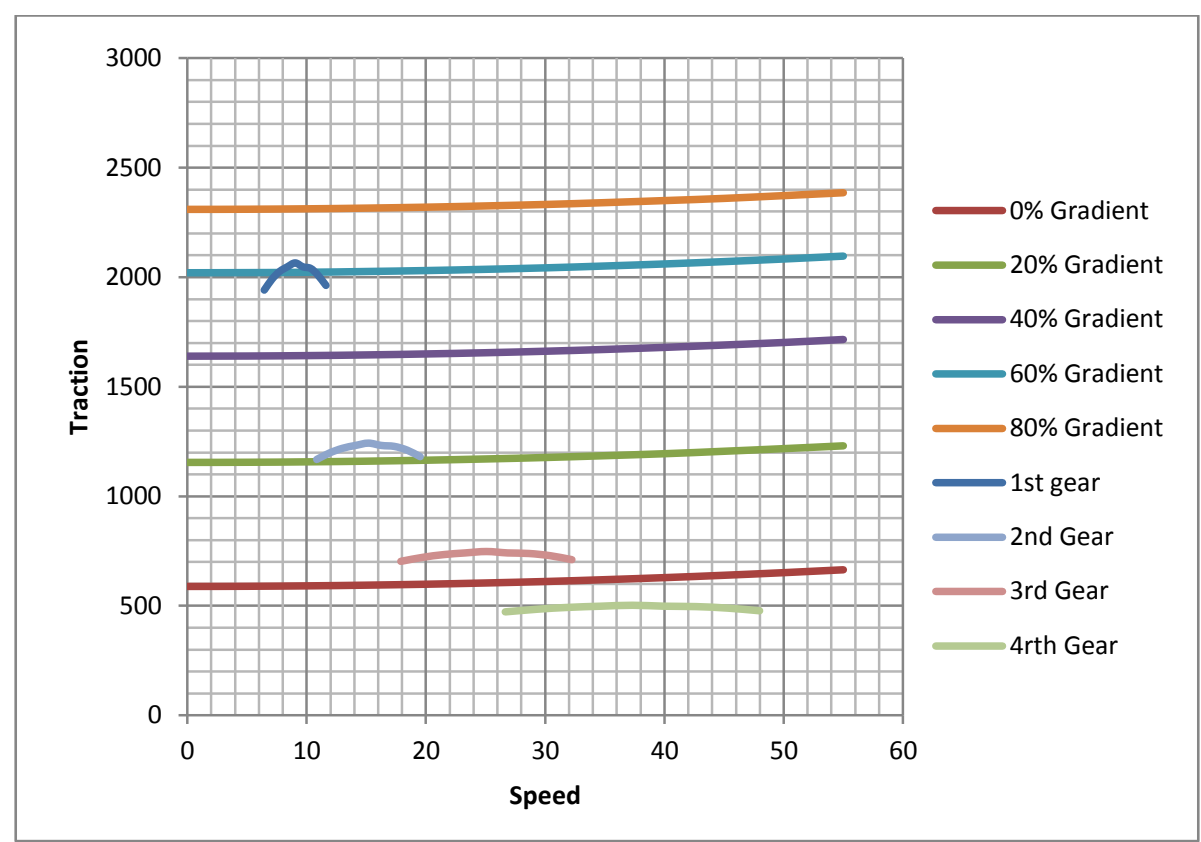

\subsection{Results}

Fig. 2

The gradeability can be calculated from the above graph, which is $64 \%$ for this vehicle.

\section{References}

[1] Fundamentals of Vehicle Dynamics by Thomas D. Gillespie, Society of Automotive Engineers.

[2] Automotive Transmissions Fundamentals, Design and Applications, Gisbert Lechner and Harold Naunheimer

[3] Engine power-torque curve, http://engines.findthebest.com/l/159/Briggs-And-Stratton-1450-14-50-ft-lbs-Gross-Torque 